\title{
Re: Role of dexmedetomidine as adjuvant in postoperative sciatic popliteal and adductor canal analgesia in trauma patients: a randomized controlled trial
}

\author{
Ki-Jae Lee ${ }^{1}$ and A Ram Doo ${ }^{1,2}$ \\ 'Department of Anesthesiology and Pain Medicine, Jeonbuk National University Medical School, Jeonju, Korea \\ ${ }^{2}$ Research Institute of Clinical Medicine of Jeonbuk National University-Biomedical Research Institute of Jeonbuk National University Hospital, \\ Jeonju, Korea
}

Received April 3, 2020, Revised May 8, 2020, Accepted May 28, 2020

Handling Editor: Jee Youn Moon

Correspondence

Ki-Jae Lee

Department of Anesthesiology and Pain Medicine, Jeonbuk National University Hospital, 20 Geonji-ro, Deokjin-gu, Jeonju 54907, Korea

Tel: +82-63-250-1241, Fax: +82-63-250-1240, E-mail: lovingij1@gmail.com

\section{TO THE EDITOR}

Dexmedetomidine (DMED), a highly selective alpha-2 adrenergic agonistic agent, is one of the preferred sedatives due to its outstanding characteristics including sympatholytic, sedative, hypnotic, and analgesic efficacy [1]. And it can achieve an appropriate level of sedation without respiratory depression. Especially, the efficacy of dexmedetomidine when combined with regional anesthesia includes increasing the quality of regional anesthesia, prolongation of the duration of analgesia, and having an opioid-sparing effect postoperatively [2].

We have carefully read with great concern the article entitled "Role of dexmedetomidine as adjuvant in postoperative sciatic popliteal and adductor canal analgesia in trauma patients: a randomized controlled trial." published in The Korean Journal of Pain by Ahuja et al. [2]. Their results showed that perineurally or intravenously administered dexmedetomidine reduced postoperative tramadol consumption in patients undergoing lower extremity sur- gery when combined with sciatic popliteal and adductor canal analgesia. And they reported that hemodynamic parameters were within the normal physiologic range during the 48 hours of follow-up, even though they didn't show the raw results. However, in clinical practice, the patients who received intravenous dexmedetomidine often experience hypotension or bradycardia, even postoperatively. As investigated in a few studies, the hemodynamic effects of dexmedetomidine such as hypotension and bradycardia are well known in the perioperative period, as well as in intensive care unit (ICU) settings [3-6].

We are working on ways to identify the incidence and risk factors for dexmedetomidine-induced hemodynamic instability in perioperative settings. We conceived that body composition, such as fat percentage to total body weight, is one of the contributing factors, which can affect the volume of distribution of the drug, because dexmedetomidine is a highly lipophilic drug [7]. Indeed, in the clinical practice, we are often faced with the overdosing of anesthetic drugs with high lipophilicity by dosing based (c) This is an open-access article distributed under the terms of the Creative Commons Attribution Non-Commercial License (http://creativecommons.org/licenses/by-nc/4.0/), which permits unrestricted non-commercial use, distribution, and reproduction in any medium, provided the original work is properly cited.

(c) The Korean Pain Society, 2020
Author contributions: Ki-Jae Lee: Writing/manuscript preparation; A Ram Doo: Supervision. 
on total body weight, especially in female patients having a high body mass index.

The dosing scheme of dexmedetomidine may be modified in the susceptible populations. Advancing the quality of care and patient safety could be achieved by individualized anesthetic and risk management. Moreover, safety concerns regarding perineural administration of dexmedetomidine, such as neurotoxicity, should be further investigated in future studies.

\section{CONFLICT OF INTEREST}

No potential conflict of interest relevant to this article was reported.

\section{FUNDING}

No funding to declare.

\section{ORCID}

Ki-Jae Lee, https://orcid.org/0000-0002-2591-455X

A Ram Doo, https://orcid.org/0000-0003-1310-790X

\section{REFERENCES}

1. Belleville JP, Ward DS, Bloor BC, Maze M. Effects of intravenous dexmedetomidine in humans. I. Sedation, ventilation, and metabolic rate. Anesthesiology 1992; 77: 1125-33.

2. Ahuja V, Thapa D, Chander A, Gombar S, Gupta R, Gupta S. Role of dexmedetomidine as adjuvant in postoperative sciatic popliteal and adductor canal analgesia in trauma patients: a randomized controlled trial. Korean J Pain 2020; 33: 166-75.

3. Jakob SM, Ruokonen E, Grounds RM, Sarapohja T, Garratt C, Pocock SJ, et al. Dexmedetomidine vs midazolam or propofol for sedation during prolonged mechanical ventilation: two randomized controlled trials. JAMA 2012; 307: 1151-60.

4. Riker RR, Shehabi Y, Bokesch PM, Ceraso D, Wisemandle W, Koura F, et al. Dexmedetomidine vs midazolam for sedation of critically ill patients: a randomized trial. JAMA 2009; 301: 489-99.

5. Gerlach AT, Dasta JF, Steinberg S, Martin LC, Cook CH. A new dosing protocol reduces dexmedetomidine-associated hypotension in critically ill surgical patients. J Crit Care 2009; 24: 568-74.

6. Klinger RY, White WD, Hale B, Habib AS, Bennett-Guerrero E. Hemodynamic impact of dexmedetomidine administration in 15,656 noncardiac surgical cases. J Clin Anesth 2012; 24: 212-20.

7. Weerink MAS, Struys MMRF, Hannivoort LN, Barends CRM, Absalom AR, Colin P. Clinical pharmacokinetics and pharmacodynamics of dexmedetomidine. Clin Pharmacokinet 2017; 56: 893-913. 